\title{
Lab automation
}

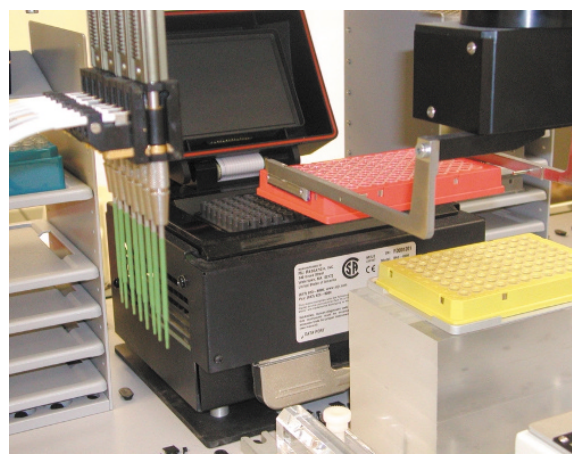

\section{Gene cloning}

Pangene's Gene Gin combines robotics, magnetic separation, and the company's gene isolation technologies to make possible high-throughput gene cloning of thousands of full-length genes for use in applications including drug target discovery for target biovalidation, pharmacogenomics, and personalized medicines. Genomic and cDNA can be isolated without using PCR in the gene cloning process, which uses DNA repair enzyme-mediated processes to capture homologous and/or nearly homologous DNA clones from complex DNA mixtures.

http://www.pangene.com

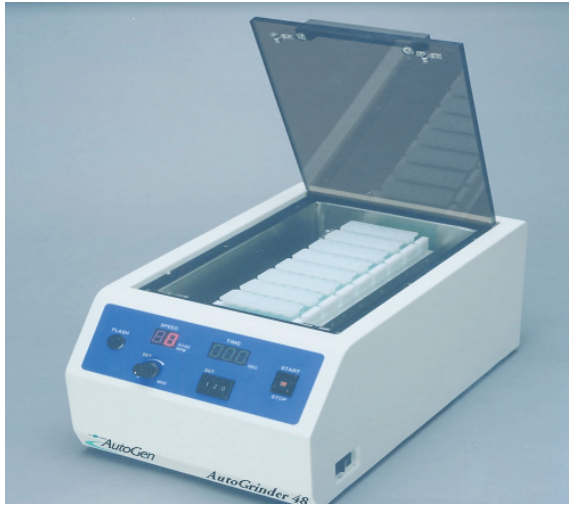

\section{Plant DNA extraction}

AutoGen introduces the AutoGrinder 48 for complete automation of plant sample grinding. Users simply place their plant material and a few zirconium beads into AutoGen tube units, freeze them, and place them onto the AutoGrinder. The entire grinding process is completed in just $2 \mathrm{~min}$ for up to 48 samples. When combined with one of the AutoGenprep systems for automated plant DNA extraction, users have a fully automated system at the lowest cost per prep with consistently high yield. http://www.autogen.com
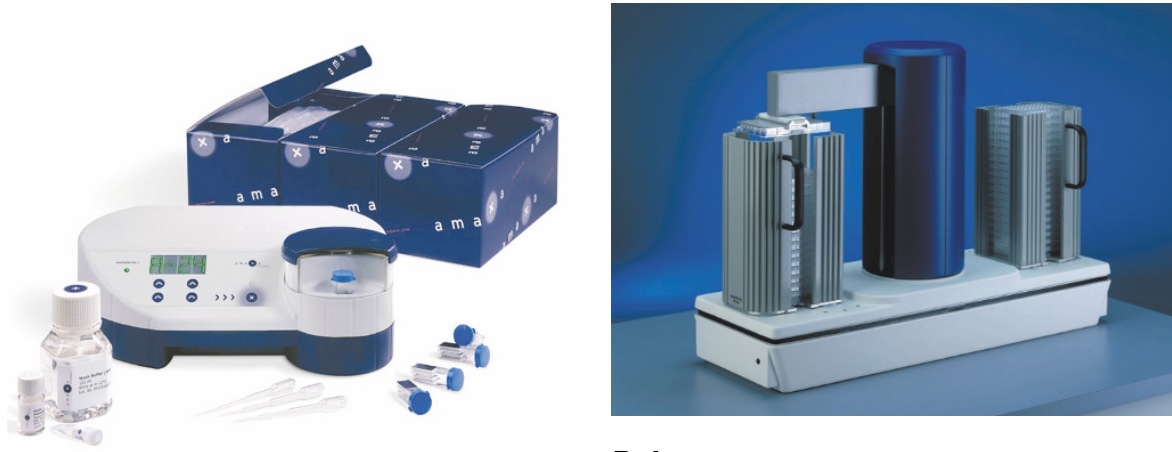

\section{Robot arm}

\section{Gene transfer}

Amaxa Biosystems' Nucleofector technology is the first nonviral gene transfer method that achieves high gene transfer efficiencies into primary cells and hard-to-transfect cell lines. DNA is directly transferred into the cell's nucleus, therefore highly efficient gene transfer can be obtained even in unstimulated and nondividing cells. The technology uses a combination of electrical parameters, delivered by the Nucleofector device, and cell type-specific Nucleofector solutions. Amaxa currently provides ready-to-use protocols for 10 different primary cell types, such as dermal and endothelial cells as well as blood and smooth muscle cells.

http://www.amaxa.com

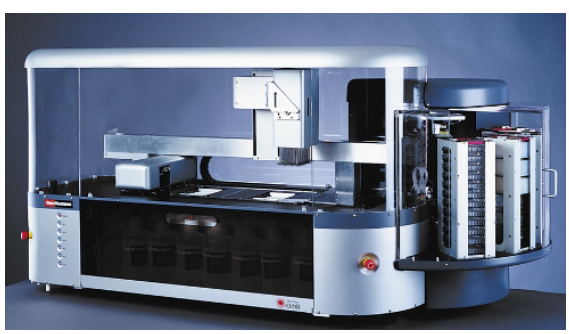

\section{Sample prep workstation}

The RevPrep Orbit workstation from GeneMachines is a cost-effective solution for plasmid purification, with a cost per sample of less than $\$ 0.10$ with sequence quality equal to or better than filter-based methods. Featuring unique Array Centrifuge technology, composed of 96 separate rotors with microwell-plate spacing that function as both sample wells and miniature centrifuges, a 96-channel pipetter, an 8-reagent bulk dispenser, a Server Arm automated plate handler, and 48-plate storage capacity, the workstation's throughput is 12 plates $(>1,100$ samples) in $8 \mathrm{~h}$, from cell culture to purified DNA, completely unattended. http://www.genemachines.com
QIAGEN's BioRobot Twister I robotic arm systems integrate with BioRobot workstations and a wide range of external instruments, such as spectrophotometers, plate sealers, thermal cyclers, and plate storage devices, for fully automated processing of serial applications. BioRobot Twister I software is fully compatible with QIAsoft, the BioRobot Operating System software, ensuring a fast startup and centralized process control.

http://www.qiagen.com

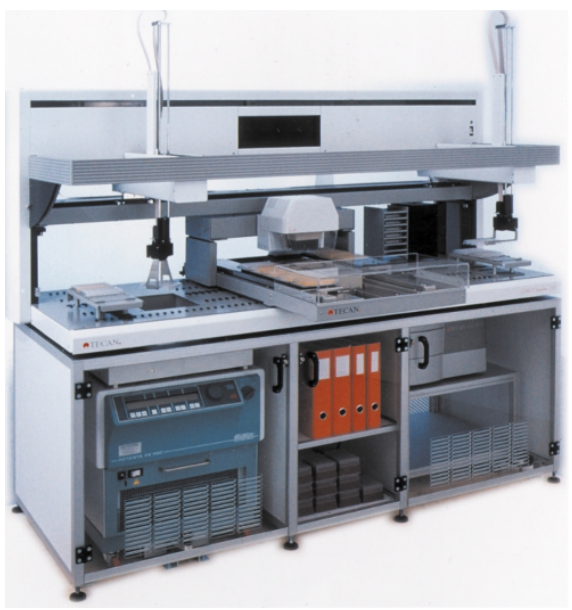

High-throughput system

The Genesis Freedom workstation is a modular, scalable, high-throughput system that allows researchers to integrate and automate complex genomics, proteomics, and drug discovery research processes. Accepting up to three robotic arms, nine different arm configurations, and a wide selection of manipulators and accessories, the workstation accelerates processes such as nucleic acid extraction and sample prep, PCR setup, DNA normalization, genotyping, SNP scoring, and in situ hybridization for high-throughput gene expression and functional studies.

http://www.tecan.com 